\title{
Peer-Led Education Expedites Deprescribing Proton Pump Inhibitors for Appropriate Veterans
}

\begin{abstract}
Costly proton pump inhibitors have been widely prescribed since the 1990 s for prevention and treatment of ulcers and gastroesophageal reflux disease. Evidence published since 2012 demonstrates risks associated with taking proton pump inhibitors for longer than 8 weeks. Primary care providers mostly deprescribe proton pump inhibitors for persons not meeting criteria for long-term use. Many patients resist discontinuation.

A 3-month evidence-based practice education project was conducted by a nurse practitioner to improve primary care provider peer deprescribing successes with appropriate patients in an outpatient California-based veteran primary care clinic. Fifteen primary care providers were pretested about usual care practices between 2 comparable clinics. Five primary care providers at the smaller clinic location were educated about long-term proton pump inhibitor use risks and introduced to 3 evidence-based practice guidelines using tapering techniques with follow-up care. A Canadian 2017 evidence-based practice proton pump inhibitor deprescribing guideline was proposed for translation into practice. Primary care providers voted to pilot this guideline, dependent upon nursing support. Primary care providers denied frustration with usual care practices, even as all were willing to try an evidence-based practice change between pre- and post-test surveys. Support for peer-led evidence-based practice on-site coaching increased from $87 \%$ to $100 \%$. Tapering behavior increased from $67 \%$ to $100 \%$, expediting improved long-term medication cessation.
\end{abstract}

$\mathrm{P}$ roton pump inhibitors (PPIs), first used in 1989, attained regulatory approval as safe for long-term use to prevent ulcers in high-risk patients and to treat various gastrointestinal pathologies (Avraham \& Biglow, 2018). Subsequently, PPIs have been prescribed for long-term use and are one

Received February 21, 2019; accepted May 13, 2019.

About the author: Mary H. Bowman, DNP, FNP-C, APRN, is Primary Care Provider, San Diego VA HealthCare System, San Diego, California.

The content of this manuscript is the responsibility of the author alone and does not necessarily reflect the view or policies of the Department of Veterans Affairs or the United States Government.

The author declares no conflict of interest.

This is an open-access article distributed under the terms of the Creative Commons Attribution-Non Commercial-No Derivatives License 4.0 (CCBY-NC-ND), where it is permissible to download and share the work provided it is properly cited. The work cannot be changed in any way or used commercially without permission from the journal.

Correspondence to: Mary H. Bowman, DNP, FNP-C, APRN (maryhbowman57@gmail.com).

DOI: $10.1097 /$ SGA.0000000000000479 of the costliest, widely prescribed medications given to prevent and treat gastritis, laryngeal symptoms (Gatta et al., 2007), and gastroesophageal reflux disease (GERD) symptoms (Farrell et al., 2017; Naunton, Peterson, Deeks, Young, \& Kosari, 2018; Reeve et al., 2015; Thompson, Black, et al., 2018). Evidence published since 2013 supports deprescribing patients with no exclusion criteria to limit the comorbidities associated with long-term PPI use (Avraham \& Biglow, 2018; Gualtero et al., 2017; Ho et al., 2014; Khan, Ismail, Haider, \& Ali, 2018; Lazarus et al., 2016; Thompson, Black, et al., 2017; Xie et al., 2016).

\section{Background}

Longitudinal, observational cohort studies report associations between long-term PPI use and communityacquired pneumonia, Clostridium difficile infections, diarrhea, chronic renal insufficiency, headaches, hypocalcemia, osteoporotic fractures, hypomagnesemia, vitamin $\mathrm{B}_{12}$ deficiency, and QT prolongation (Farrell et al., 2017; Ho et al., 2014; Khan et al., 2018; 
Lazarus et al., 2016; Xie et al., 2016). Other associated concerns are an increased risk for developing gastric tumors and gastric carcinoma. Jianu et al. (2012) presented two case studies demonstrating hypergastrinemia secondary to PPI therapy, concluding that enterochromaffin-like carcinoids could arise from long-term PPI use. Dado, Loesch, and Jaganathan (2017) reported a case study of severe iron-deficiency anemia associated with long-term PPI use. A large cohort study of veterans by Xie et al. (2016) cited excess risk of death among those taking PPIs for a long time without necessity, when compared with those taking histamine receptor agonists (H2RAs).

According to Avraham and Biglow (2018), PPIs are inappropriately prescribed $48.59 \%$ of the time. In Australia, Reeve et al. (2015) estimated inappropriate use at $50 \%$. Thompson, Black, et al. (2017) reported that $50 \%$ of Canadian patients remained on PPIs longterm without need. In a U.S. cohort study of PPI use between 2001 and 2011, Haastrup et al. (2016) revealed high correlations between new onset of longterm PPI use and low-income/low education levels. Of these long-term users, $96 \%$ did not have a diagnosis that necessitated PPI therapy (Haastrup et al., 2016).

Exclusion criteria for deprescribing PPIs are advanced stages of GERD, Barrett's esophagitis, tumor or metastasis, mechanical ventilation, hospice or palliative care, radiation therapy or chemotherapy, pathological hypersecretory conditions, use of antiplatelet or anticoagulation therapy, variceal or gastrointestinal hemorrhages, or long-term nonsteroidal anti-inflammatory drug use (Avraham \& Biglow, 2018; Thompson, Black, et al., 2017). Patients not meeting Los Angeles (LA) Classification (Table 1) Grade C or D gastroesophageal reflux symptoms and with no history of ulcers or diseaserelated pathological gastropathies may be deprescribed from PPIs after 4-8 weeks of use (Farrell et al., 2017).

Many patients report taking PPIs for years without reason and are reluctant to cease use (Avraham \& Biglow, 2018; Farrell et al., 2017; Naunton et al., 2018;
Reeve et al., 2015; Thompson, Black, et. al., 2017; Xie et al., 2016). Thompson, Black, et al. (2017) performed a scoping review of seven survey studies, four qualitative studies, and one randomized controlled trial. One of these studies evaluated patient preferences with respect to decreasing or ceasing PPI use. Thompson, Black, et al. (2017) cited patients reporting significant value in controlling their GERD symptoms along with anxiety about rebound symptoms with PPI dose reductions. Furthermore, they found that patients held a wide range of attitudes about long-term PPI use and tapering dosages. They cautioned that sensitivity must be given to patient preferences when negotiating shared decisions to continue or taper dosing (Thompson, Black, et al., 2017). Rather than total cessation, Thompson, Black, et al. (2017) reported that appropriate patients were willing to taper to the lowest dose for symptom control. The economic savings of limiting long-term PPI use correlated to decreased side effect-related costs and medication cost reductions with as-needed use (Thompson, Farrell, et al., 2017). In a review of PPI use across international borders, Naunton et al. (2018) concluded that deprescribing efforts should be a priority, due to worldwide excess use.

\section{Deprescribing Challenges Call for Evidence-Based Practice Guideline Use}

Since 2014, gastroenterologists have routinely advised primary care providers (PCPs) to deprescribe patients without exclusion criteria after 8 weeks or less, but with minimal guideline support (Avraham \& Biglow, 2018; Farrell et al., 2017; Reeve et al., 2015). Thompson, Black, et al. (2017) reported strong evidence that few patients required therapeutic care with long-term PPI use. Deprescribing constituted multiple options: sudden abruption, tapering to half dosing and then to use as needed, or substituting H2RAs to control rebound GERD symptoms (Avraham \& Biglow, 2018; Farrell et al., 2017; Reeve et al., 2015; Thompson,

\section{TABLE 1. The Los Angeles Classification of Esophagitis}

\section{Grade Definition}

A One (or more) mucosal break no longer than $5 \mathrm{~mm}$ that does not extend between the tops of two mucosal folds

B One (or more) mucosal break more than $5 \mathrm{~mm}$ long that does not extend between the tops of two mucosal folds

C One (or more) mucosal break that is continuous between the tops of two or more mucosal folds but which involves less than $75 \%$ of the circumference

D One (or more) mucosal break which involves at least $75 \%$ of the esophageal circumference

Note. Adapted from "The Los Angeles Classification of Gastroesophageal Reflux Disease," by S. S. Sami and K. Ragunath, 2013, Video Journal and Encyclopedia of Gl endoscopy, 1, pp. 103-104. Copyright 2013 by Elsevier GmbH. Used with permission. 
Black, et al., 2017). Except for abrupt cessation, all of these methods were supported by the evidence (Avraham \& Biglow, 2018; Farrell et al., 2017; Reeve et al., 2015; Thompson, Black, et al., 2017). Notwithstanding, patients occasionally ceased longterm use of PPIs abruptly and reported symptoms of rebound acid hypersecretion (RAHS). Evidence does not support abrupt cessation (Farrell et al., 2017; Naunton et al., 2018; Reeve et al., 2015; Thompson, Black, et al., 2017), as RAHS might inhibit PPI cessation maintenance in deprescribed long-term users.

Zalvan, Hu, and Greenberg (2017) cited studies promoting standard reflux precautions with no decrease in GERD incidence. Lifestyle precautions (e.g., avoidance of heavy late-night meals, spicy and acidic foods, tobacco, alcohol and caffeine) have been promoted empirically to reduce GERD symptoms (Meining \& Classen, 2000). Lifestyle and dietary recommendations have not been found to be associated with the development, management, or progression of GERD, except in studies with small sample sizes (Meining \& Classen, 2000).

Ness-Jensen, Hveem, El-Serag, and Lagergren (2016) performed a systematic review of the evidence to update lifestyle and dietary recommendations helpful in GERD symptom management. Two randomized controlled trials suggested weight loss and tobacco cessation to manage GERD and also cited avoidance of late-night meals and head elevation in bed as effective for managing nocturnally occurring GERD symptoms (Ness-Jensen et al., 2016). Only these four lifestyle changes were found to be effective for managing GERD in the evidence (Ness-Jensen et al., 2016).

Primary care providers may rely on experience in choosing deprescribing methods. The use of deprescribing guidelines could help patients share in decision making for tapering and cessation to improve sustained outcomes (Thompson, Black, et al., 2017). Prescribers must consider patient values and preferences (Thompson, Black, et al., 2017; Thompson, Farrell, et al., 2017).

\section{Identification of Site-Specific Need for Evidence-Based Guideline Use}

In a California-based Veterans Administration (CVA) primary care (PC) clinic population, the usual deprescribing care for appropriate patients is performed by PCPs. Proton pump inhibitors are typically deprescribed after 4-8 weeks of use, per institutional pharmacy recommendations implemented in 2015. Primary care providers globally advise patients to seek followup if their GERD symptoms return. Tapering and substitution with formulary H2RAs are recommended without parameter specifications.

Problems with the usual care occur without structured follow-up. Patients may abruptly cease use of PPIs and experience RAHS and resume them by purchasing them over the counter. CVA PCPs are not specifically guided to seek testing for Helicobacter pylori antibodies or to order endoscopies after deprescribing patients who report ongoing GERD symptoms following deprescribing failures. Farrell et al. (2017) recommend selection for exclusion of deprescribing be made for patients known to have L.A. Classification C or D GERD, with or without a history of ulcers. Farrell et al. (2017) also recommend in their PPI deprescribing guideline that patients failing deprescribing be tested for Helicobacter pylori antibodies.

CVA PCPs are not guided to deprescribe PPIs according to the breadth of exclusion criteria recommended in other guidelines. Two patient care instruction sheets are provided for PCPs to give to patients, which discuss overutilization of PPIs and associated risks and offer dietary guidance with foods to choose and avoid to manage GERD. The food lists are not supported by current evidence to reduce GERD symptoms (Ness-Jensen et al., 2016). Patients report resuming PPIs due to their efficacy, even with risks for many long-term users (Farrell et al., 2017; Naunton et al., 2018; Thompson, Black, et al., 2017). Follow-up mechanisms to reassess patients at intervals following deprescribing are supported by newer evidence to expedite sustained cessation of use (Farrell et al., 2017; Naunton et al., 2018). Patients who continue to take PPIs after deprescribing may forgo timely endoscopies and biopsies, which diagnose Helicobacter pylori antibodies, Barrett's esophagitis, or esophageal cancer.

\section{Three PPI Deprescribing Guidelines Found in the Evidence for Translation}

New protocols recommend use of specific objective criteria for deprescribing PPIs with tapering choices and close follow-up care. In their pilot study of 10 elderly nursing home residents, Avraham and Biglow (2018) developed a guideline for PPI deprescribing by reducing the dose in half every 3 weeks and then changing the frequency to every-other-day use over 3 weeks. Followup assessments were recommended by a clinical pharmacist every 3 weeks during deprescribing over the course of 12 weeks (Avraham \& Biglow, 2018). This guideline requires four follow-up visits or phone call assessments during tapering (Avraham \& Biglow, 2018).

In 2013, Reeve et al. (2015) developed a PPI deprescribing guideline in Australia. Fifty-seven PPI users were recruited in this feasibility study; six participated and achieved successful cessation for a minimum of 6 months. Tapering was recommended by reducing PPI dosing in half using a symptom action plan to add doses as needed or to return to the prior dose to alleviate onset of associated, severe RAHS during deprescribing (Reeve et al., 2015). Ten-minute pharmacy-led interventions by phone occurred every 2 weeks during the 6 weeks of 
deprescribing; additional phone calls were made 6 weeks and 6 months after deprescribing was completed for evaluation (Reeve et al., 2015). On average, 4.3 phone calls were made to the six participants (Reeve et al., 2015). Health professionals spent 1.5 hours per deprescribed patient (Reeve et al., 2015).

Farrell et al. (2017) constructed an evidence-based PPI deprescribing guideline with multidisciplinary input from experts in Canada. Development was based on data from meta-analyses of randomized controlled trials and primarily from cohort studies. Farrell et al.'s (2017) PPI deprescribing guideline aims to improve effective PPI deprescribing with simple, tapering instructions and follow-up care to mitigate patient safety risks. It was developed with specific GRADE (Grading of Recommendations, Assessment, Development, and Evaluation) criteria (Farrell et al., 2016) and incorporates use of the L.A. Classification of GERD for international use (Farrell et al., 2017).

First developed in 1994, the LA Classification of GERD was supported in 1999 by the World Organization of Gastroenterology after it was presented at the Los Angeles World Congress of Gastroenterology (Table 1). This classification system uses endoscopic findings and circumferential measurements to objectively determine the severity of GERD by enumerating and measuring erosions in mucosal breaks of greater than or less than $5 \mathrm{~mm}$ in size and the degree of erosions between the tops of two mucosal folds (Lundell et al., 1999; Sami \& Ragunath, 2013). Use in clinical decision-making for deprescribing may ensure that patients with severe GERD are not inappropriately deprescribed PPIs. Lundell et al. (1999) and Sami and Ragunath (2013) confirmed that the L.A. Classification of GERD remains the most widely used tool for classification of GERD severity.

Farrell et al. (2017) recommend against deprescribing for patients known to have L.A. Classification C or D GERD, regardless of a history of ulcers. Farrell et al. (2017) also recommend in their PPI deprescribing guideline that patients be tested for Helicobacter pylori antibodies if they initially fail PPI deprescribing. Avraham and Biglow's (2018) deprescribing guideline does not incorporate the L.A. Classification of GERD in clinical decision-making that excluded inappropriate persons.

\section{Peer-Led Education as an Intervention to Support Translation}

Peer-led education is supported in the evidence for practice changes. Ip et al.'s (2013) longitudinal study reported that mandatory peer-to-peer consultation in radiology medical management reduced costs by limiting inappropriate use of high-cost imaging studies. A separate scoping review of four studies evaluated the efficacy of peer-led education in improving patient safety (Walpola, McLachlan, \& Chen, 2018). Teams in PC utilizing training of peer-to-PCPs (i.e., MDs, nurse practitioners [NPs]) and peer-to-registered nurses (RNs) were able to promote improving deprescribing practices.

\section{Description of Evidence-Based Practice Project}

The goal of this quality improvement (QI) education project was to attain consensus from CVA PCPs to try to implement a new PPI deprescribing algorithm into practice for appropriate veterans in a PC population. This project was approved by the CVA's institutional review board for the project site where PCPs were surveyed and educated about newer PPI deprescribing guidelines. A second nearby CVA PC location was also approved for the administration of a pretest survey for PCPs who were not to be included as participants in the education project for the purpose of collecting input about PCP satisfaction with the usual care.

A PCP peer (NP employed full-time on-site) presented three evidence-based PPI deprescribing guidelines to PCPs at one practice site after PCPs in two similar CVA PC practices completed a 12-question Likert-type survey as a pretest measure of usual care for deprescribing PPIs $(n=15)$. At the project site, provider willingness to change deprescribing care practices was assessed in three peer-led, 45-minute education sessions, 4 weeks apart, over the 12 weeks of the study with a convenience sample of five PCPs. Four board-certified internal medicine physicians and one board-certified master's prepared NP comprised the participant group. After 12 weeks, the Likert-type survey was again administered as a post-test survey for the five PCP participants who completed education.

Selected articles discussing the side effects of chronic PPI use and problems with abrupt cessation of use were disseminated to the participating PCPs (Farrell et al., 2017; Nehra, Alexander, Loftus, \& Nehra, 2018; Reeve et al., 2015; Thompson, Black, et al., 2017). Three PPI evidence-based deprescribing guidelines (Avraham \& Biglow, 2018; Farrell et al., 2017; Reeve et al., 2015) were introduced for critical evaluation. Each guideline recommended tapering PPIs prior to cessation and the intervals for follow-up of patients to ascertain cessation maintenance. Farrell et al.'s (2017) PPI deprescribing guideline required the fewest team-driven callbacks $(n=2)$ at 4and 12-week intervals following PPI deprescribing.

\section{Results}

Primary care providers agreed by consensus to pilot implementation of Farrell et al.'s (2017) PPI deprescribing guideline following 12 weeks of intermittent education dependent upon staff availability for callbacks. Farrell et al.'s (2017) PPI deprescribing guideline was perceived 


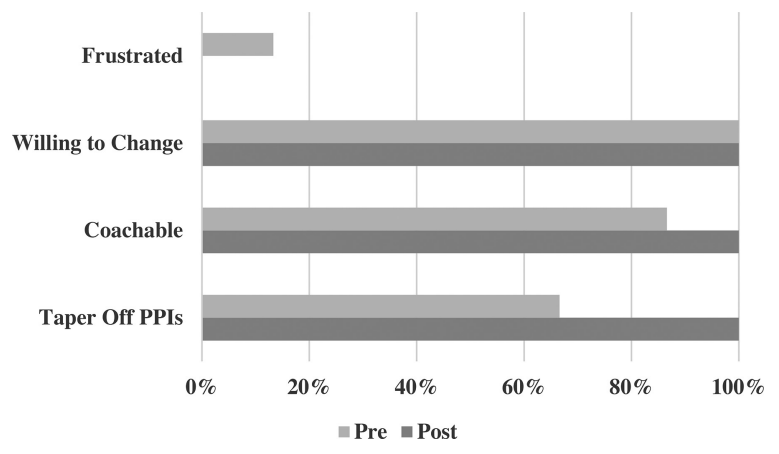

FIGURE 1. Pre- and post-test survey results and changes as a percentage of total responses. $\mathrm{PPI}=$ proton pump inhibitor.

to represent best care practices using L.A. Classification of GERD criteria, was cited to have been developed with GRADE, and was more feasible to implement than other guidelines requiring additional phone call follow-up.

Support from the Department of Pharmacy for patient follow-up calls was not available. The NP peer surveyed the PC RNs' interest in participating in a pilot study and willingness to call patients at 4- and 12-week intervals following deprescribing. A tool that had been used in other evidence-based projects was discussed for translation for RNs to use to support PPI cessation maintenance after tapering. Of the seven RNs providing feedback, all RNs expressed interest in supporting the project. Limited time and conflicting responsibilities were cited as barriers to RN participation. One RN offered to provide support to all five PCPs who approved of a pilot of the preferred guideline. She offered to help after hours, toward meeting a promotion requirement.

Most PCPs acknowledged in pretesting that up to one third of their patients were taking PPIs for a long term after deprescribing. Two of 15 PCPs who were pretested $(13.3 \%)$ were frustrated with these results; yet, all were willing to implement an evidence-based practice (EBP) guideline to improve care. Most PCPs $(83 \%)$ supported peer-led on-site education and coaching to assist with translation of EBP guideline use into practice. In total, $66.6 \%$ of the providers reported the need to taper off PPIs. By the postintervention survey, the five volunteer PCPs surveyed were in complete agreement on the four areas of the survey: Frustration (none), willingness to change, coachability, and tapering behaviors increased to $100 \%$ (Figure 1). Results lacked statistical power for generalizability in the postintervention survey because five of the 15 surveyed PCPs participated in the survey following the education intervention provided at the smallest local clinic site.

Primary care providers at the PC education site explained that, following education, they had some uncertainty as to the value of a pilot launch to test the guideline due to limited resources for callbacks from staff for support and the perception that they had already begun deprescribing PPIs with tapering strategies according to Farrell et al.'s (2017) PPI deprescribing guideline. They indicated that they might not find enough appropriate remaining patients left on their panels to deprescribe according to the guideline in a pilot study. Furthermore, burnout of PCPs at this VA network was reported as high by PCPs in concurrent separate surveys and discussions. Primary care providers indicated their performance measurements were based on answering to national benchmark clinical reminder satisfaction data driving care prioritization they had no control over at local sites. Opioid deprescribing was cited by PCPs as a nationally driven measured performance priority.

The "Four Levels," established in a dissertation by Donald Kirkpatrick in 1959, and as expanded upon by Kirkpatrick and Kirkpatrick (2016), were used to ascertain the quality of this QI project. Level II changes were discovered between pre- and post-test results, specific to reported changes in PCP tapering behaviors. Level III behavior changes were cited. A physician indicated he tapered himself off long-term PPI use, subsequent to QI education. Two PCPs provided Level III evaluations of the efficacy of the education by the NP peer when they explained they were already tapering in their deprescribing practices in accordance with Farrell et al.'s (2017) PPI deprescribing guideline and therefore might not find enough subjects for a pilot study.

The NP peer (author) presented a poster with costs for the education and improved deprescribing results shown via Kirkpatrick and Kirkpatrick's (2016) "Four Levels" with use of Farrell et al.'s (2017) PPI deprescribing guideline, following completion of the education project, to all stakeholders. The poster displayed historical PPI prescribing patterns, problems resulting from long-term use, and goals for reduction of risks for side effects as driving the need for practice changes and projected cost savings for improved deprescribing care. A pilot launch was abandoned, as resources were not available for nursing or pharmacy support for

TABLE 2. Staff Education Cost Analysis

\begin{tabular}{|l|c|c|c|}
\hline Payroll & Quantity & Rate & $\begin{array}{c}\text { Total } \\
\text { Cost }\end{array}$ \\
\hline RN & 7 & $\$ 50.00 \times 2.5 \mathrm{hr}$ & $\$ 875$ \\
\hline $\mathrm{NP}$ & 1 & $\$ 70.00 \times 2.5 \mathrm{hr}$ & $\$ 175$ \\
\hline $\mathrm{MD}$ & 4 & $\$ 95.00 \times 2.5 \mathrm{hr}$ & $\$ 950$ \\
\hline Total wages & 12 & & $\$ 2,000$ \\
\hline Supplies & 100 copies & $\$ 0.10$ each & $\$ 10$ \\
\hline Grand total & & & $\$ 2,010$ \\
\hline
\end{tabular}


follow-up calls to be added to staff workloads, given demands of higher system-wide priorities cited by staff.

Education costs were ascertained by estimating average salaries of four attending physicians, one NP, and seven RNs educated in three 45-minute routine monthly staff meetings, which they were already required to attend for performance improvement (Table 2).

The only other cost was for copying articles to disseminate among the providers. Prescription cost savings to the organization and to patients who paid copays or over the counter for PPIs were presumed extensive and not quantifiable within the scope of this project. Decreases in acute care visits for complications from long-term PPI use were not measured within the scope of this project.

\section{Project Impact and Sustainability}

Nurse practitioner peer-led education to assist PCPs in deprescribing PPI practice changes in one CVA PC clinic may promote practice changes at other PC sites extending outside this healthcare system. Evidence was disseminated at a statewide NP education conference in a second poster presentation. Presentations that promote peer-led education to assist with selection of the most appropriate EBP guideline for site-specific use may be useful for integration of practice changes into application. Kirkpatrick and Kirkpatrick's (2016) "Four Levels" may be utilized to ascertain the quality of similar education projects. Impact and sustainability may be higher in other organizations that can be educated to PPI deprescribing guideline use at national poster conferences. Nationally driven oversight of performance measures is predicated for providers in the VA Healthcare System, and this prioritization was found to impede EBP changes requiring full interdisciplinary integration of this guideline with callbacks to patients. The VA has not cited PPI deprescribing as a priority in PC clinical reminder reporting for benchmark care satisfaction data analysis. Success of future VA site-specific EBP projects may depend on choosing projects that address problems already identified as national clinical priorities to ensure nursing staff support for integration.

Pursuant to peer-led education at this site, PCPs were uniformly willing to pilot a study to change practice to improve care, if staff support were available, even as high rates of PCP burnout were reported system-wide during the project. Provider frustration was not found to drive willingness to change from the usual care. A majority of PCPs acknowledged in pretest surveys they cared for patients using PPIs for a long term who qualified to discontinue use; one third of PCPs were not aware to implement tapering for sustained cessation. Future research could evaluate factors other than provider frustration that promote openness to practice changes.

\section{Strengths and Limitations}

The education intervention was partly successful, even without a pilot launch, because PCPs reported higher tapering practice changes found in the evidence to be more successful for long-term PPI cessation, which constituted a Level III behavior change (Kirkpatrick \& Kirkpatrick, 2016). Implementing the EBP guideline to change practice was met with a major obstacle. Primary care providers did not have staff support for interval patient callbacks at this site. Primary care providers reported perceptions of high burnout from addressing other priorities under VA national guideline oversight and cited they were tasked to meet prescribed benchmark clinical reminder satisfaction for activities, such as opioid deprescribing, to uphold as priorities for performance measures. Bureaucratic oversight inhibited a local clinic to autonomously address a grassroots effort change because limited staff resources were directed toward other previously assigned national priorities.

\section{Conclusion}

The NP as a PCP peer holds a dual role of advanced practice RN and PCP to promote practice changes by providing education and coaching to both providers and staff RNs. Primary care NPs and gastroenterology (GI) nurses caring for patients in endoscopy suites may be best positioned to initiate interdisciplinary practice changes among three professional groups (i.e., MDs, NPs, and RNs). Each may serve in peer-educator roles for promoting deprescribing PPIs for appropriate patients. Gastroenterology nurses, as experts who understand LA Classification of Esophagitis, can initiate patient education for deprescribing PPIs with GI specialist input immediately following endoscopies. Gastroenterology nurses may promote peer-led education and patient education about the importance of being open to tapering off PPIs in appropriate situations, which may pave the way for PCP follow-up after endoscopies. Careful consideration of priorities supporting EBP projects may determine the success of project integration in larger organizations in PC. Identification of sites responsive to nationally driven preset practice priorities and assessment of the coexistence of PCP burnout are essential when seeking PC nursing support for practice changes.

\section{REFERENCES}

Avraham, O., \& Biglow, M. (2018). Implementation of proton pump inhibitor deprescription protocol in geriatric residents. The Annals of Pharmacotherapy, 52(8), 747-753. doi:10.1177/1060028018759747

Dado, D. N., Loesch, E. B., \& Jaganathan, S. P. (2017). A case of severe iron deficiency anemia associated with long-term proton pump inhibitor use. Current Therapeutic Research, 84, 1-3. doi:10.1016/j.curtheres.2017.01.003 
Farrell, B., Pottie, K., Rojas-Fernandez, C. H., Bjerre, L. M., Thompson, W., \& Welch, V. (2016). Methodology for developing deprescribing guidelines: Using evidence and GRADE to guide recommendations for deprescribing. PLoS One, 11(8), e0161248. doi:10.1371/journal.pone.0161248

Farrell, B., Pottie, K., Thompson, W., Boghossian, T., Pizzola, L., Rashid, F. J., ... Moayyedi, P. (2017). De-prescribing proton pump inhibitors. Canadian Family Physician, 63, 354-364. Retrieved from http://www.cfp.ca/content/cfp/63/5/354.full.pdf

Gatta, L., Vaira, D., Soorenti, G., Zucchini, S., Sama, C., \& Vakil, N. (2007). Meta-analysis: The efficacy of proton pump inhibitors for laryngeal symptoms attributed to gastro-oesophageal reflux disease. Alimentary Pharmacology \& Therapeutics, 25, 385-392. doi:10.1111/j.1365-2036.2006.03213.x

Gualtero, S. M., Abril, L. A., Camelo, N., Sanchez, S. D., Arias, G., Silva, E., ... Pareja, M. J. (2017). [Characteristics of Clostridium difficile infection in a high complexity hospital and report of the circulation of the NAP1/027 hypervirulent strain in Columbia]. Biomedica, 37, 466-472. doi:10.7705/biomedical.v37i4.3244

Haastrup, P. F., Paulsen, M. S., Christensen, R. D., Søndergaard, J., Hansen, J. M., \& Jarbøl, D. E. (2016). Medical and non-medical predictors of initiating long-term use of proton pump inhibitors: A nationwide cohort study of first-time users during a 10-year period. Alimentary Pharmacology \& Therapeutics, 44, 78-87. doi:10.1111/apt.13649

Ho, S.-W., Tsai, M.-C., Teng, Y.-H., Yeh, Y.-T., Wang, Y.-H, Yang, S.-F, \& Yeh, C.-B. (2014). Population-based cohort study on the risk of pneumonia in patients with non-traumatic intracranial hemorrhage who use proton pump inhibitors. BMJ Open, 4, e006710. doi:10.1136/bmjopen-2014-006710

Ip, I. K., Schneider, L., Seltzer, S., Smith, A., Dudley, J., Menard, A., \& Khorasani, R. (2013). Impact of provider-led technology-enabled radiology management program on imaging. The American Journal of Medicine, 126, 687-692. doi:10.1016/. amjmed.2012.11.034

Jianu, C. S., Fossmark, R., Viset, T., Qvigstad, G., Sørdal, Ø., Marvik, R., \& Waldum, H. L. (2012). Gastric carcinoids after longterm use of a proton pump inhibitor. Alimentary Pharmacology \& Therapeutics, 36, 644-649. doi:10.1111/apt.12012

Khan, Q., Ismail, M., Hiader, I., \& Ali, Z. (2018). Prevalence of the risk factors for QT prolongation and associated drug-drug interactions in a cohort of medical inpatients. Journal of the Formosan Medical Association. doi:10.1016/j.jfma.2018.01.016

Kirkpatrick, J. D., \& Kirkpatrick, W. K. (2016). Kirkpatrick's four levels of training evaluation. Alexandria, VA: ATD Press.

Lazarus, B., Chen, Y., Wilson, F. P., Sang, Y., Chang, A. R., Coresh, J., \& Grams, M. E. (2016). Proton pump inhibitor use and the risk of chronic kidney disease. JAMA Internal Medicine, 176, 238-246. doi:10.1001/jamainternmed.2015.7193

Lundell, L. R., Dent, J., Bennett, J. R., Blum, A. L., Armstrong, D., Galmiche, J. P., ... Wallin, L. (1999). Endoscopic assessment of oesophagitis: Clinical and functional correlates and further validation of the Los Angeles classification. Gut, 45, 172-180. Retrieved from https://www.ncbi.nlm.nih.gov/pmc/articles/ PMC1727604/pdf/v045p00172.pdf

Meining, A., \& Classen, M. (2000). The role of diet and lifestyle measures in the pathogenesis and treatment of gastroesophageal reflux disease. American Journal of Gastroenterology, 95, 2692-2697.

Naunton, M., Peterson, G. M., Deeks, L. S., Young, H., \& Kosari, S. (2018). We have had a gutful: The need for deprescribing proton pump inhibitors. Journal of Clinical Pharmacy and Therapeutics, 43, 65-72. doi:10.111/jcpt.12613

Nehra, A. K., Alexander, J. A., Loftus, C. G., \& Nehra, V. (2018). Proton pump inhibitors: Review of emerging concerns. Mayo Clinic Proceedings, 93, 240-246. doi:10.1016/j.mayocp.2017. 10.022

Ness-Jensen, E., Hveem, K., El-Serag, H., \& Lagergren, J. (2016). Lifestyle intervention in gastroesophageal reflux disease. Clinical Gastroenterology and Hepatology, 14, 175-182. doi:10.1016/j. cgh.2015.04.176

Reeve, E., Andrews, J. M., Wiese, M. D., Hendrix, I., Roberts, M. S., \& Shakib, S. (2015). Feasibility of a patient-centered deprescribing process to reduce inappropriate use of proton pump inhibitors. The Annals of Pharmacotherapy, 49, 29-38. doi:10.1177/1060028014558290

Sami, S. S., \& Ragunath, K. (2013). The Los Angeles classification of gastroesophageal disease. Video Journal and Encyclopedia of GI Endoscopy, 1, 103-104. doi:10.1016/S22120971(13)70046-3

Thompson, W., Black, C., Welch, V., Farrell, B., Bjerre, L. M., \& Tugwell, P. (2017). Patient values and preferences surrounding proton pump inhibitor use: A scoping review. The Patient, 11, 17-28. doi:10.1007/s40271-017-L0258-4

Thompson, W., Farrell, B., Welch, V., Tugwell, P., \& Bjerre, L. M. (2017). Should I continue taking my acid reflux medication? Design of a pilot before/after study evaluating a patient decision aid. Canadian Pharmacists Journal, 150, 19-23. doi:10.1177/1715163516679425

Walpola, R. L., McLachlan, A. J., \& Chen, T. F. (2018). A scoping review of peer-led education in patient safety training. American Journal of Pharmaceutical Education, 82(2), 6110. doi:10.5688/ ajpe 6110

Xie, Y., Bowe, B., Li, T., Xian, H., Yan, Y., \& Al-Aly, Z. (2016). Risk of death among users of proton pump inhibitors: A longitudinal observational cohort study of United States veterans. BMJ Open, 7, e015735. doi:10.1136/bmjopen-2016-015735

Zalvan, C., Hu, S., \& Greenberg, B. (2017). A comparison of alkaline water and Mediterranean diet vs. proton pump inhibition for treatment of laryngopharyngeal reflux. JAMA OtolaryngologyHead \& Neck Surgery, 143(10), 1023-1029. doi:10.1001/ jamaoto.2017.1454 